\title{
Brain white matter integrity and association with age at onset in pediatric obsessive-compulsive disorder
}

\author{
Isabelle M Rosso ${ }^{1,2^{*}}$, Elizabeth A Olson, ${ }^{1,2}$, Jennifer C Britton ${ }^{3}$, S Evelyn Stewart ${ }^{4,9}$, George Papadimitriou ${ }^{5,6,7}$, \\ William DS Killgore ${ }^{1,2}$, Nikos Makris5,6,7, Sabine Wilhelm ${ }^{2,8}$, Michael A Jenike ${ }^{2,8,10}$ and Scott L Rauch ${ }^{1,2}$
}

\begin{abstract}
Background: Obsessive-compulsive disorder (OCD) is a common and debilitating neuropsychiatric illness thought to involve abnormal connectivity of widespread brain networks, including frontal-striatal-thalamic circuits. At least half of OCD cases arise in childhood and their underlying neuropathology may differ at least in part from that of adult-onset OCD. Yet, only a few studies have examined brain white matter (WM) integrity in childhood-onset OCD using diffusion tensor imaging (DTI), and none have examined potential associations with age at onset.

Results: In this study, 17 youth with OCD and 19 healthy control subjects, ages 10 to 19 years, underwent DTI on a 3T Siemens scanner. DSM-IV diagnoses were established with standardized interviews, and OCD symptom severity was evaluated using the Children s Yale-Brown Obsessive-Compulsive Scale (CY-BOCS). Voxel-wise analyses were conducted on data processed with tract-based spatial statistics (TBSS) to derive measures of fractional anisotropy (FA), axial diffusivity (AD), radial diffusivity (RD), and mean diffusivity (MD). OCD patients had significantly lower FA in seven WM clusters, with over $80 \%$ of significant voxels in bilateral frontal cortex and corpus callosum (CC). There were no regions of significantly higher FA in patients compared with controls. Patients also had significantly higher $\mathrm{RD}$ in right frontal cortex and right body of the CC. Earlier age at onset of OCD correlated significantly with lower FA in the right thalamus and with higher RD in the right CC. FA and RD were not significantly associated with symptom severity.
\end{abstract}

Conclusions: These findings point to compromised WM integrity and reduced myelination in some brain regions of children with OCD, particularly the CC and fiber tracts that connect the frontal lobes to widespread cortical and subcortical targets. They also suggest that age at onset may be a moderator of some of the WM changes in pediatric OCD.

Keywords: Obsessive-compulsive disorder, Childhood, Diffusion tensor imaging, Corpus callosum, Thalamus, Age at onset

\section{Background}

Obsessive-compulsive disorder (OCD) is a common neuropsychiatric illness characterized by repetitive thoughts and behaviors that are unwanted, distressing, and disabling (DSM-IV; [1]). Onset occurs in childhood for more than half of OCD patients, with about $40 \%$ of pediatric cases achieving remission and the remaining

\footnotetext{
* Correspondence: irosso@hms.harvard.edu

${ }^{1}$ Center for Depression, Anxiety and Stress Research, McLean Hospital, 115 Mill Street, mailstop 334, Belmont, MA 02478, USA

2Department of Psychiatry, Harvard Medical School, Boston, MA, USA

Full list of author information is available at the end of the article
}

persisting into adulthood [2,3]. Epidemiological studies show that pediatric OCD has typical onset between 7 and 13 years [2] and that it differs from its adult-onset counterpart in a number of ways, including male preponderance, high familial loading, and more frequent comorbidity with developmental disorders $[2,4]$. Based on these correlates, it has been speculated that pediatric OCD may be a developmental subtype that is discontinuous from adult OCD, or a developmentally moderated expression of etiologic processes that are shared with the adult clinical phenotype $[5,6]$.

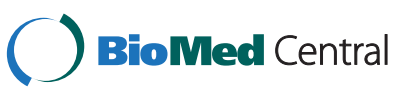

2014 Rosso et al.; licensee BioMed Central. This is an Open Access article distributed under the terms of the Creative Commons Attribution License (http://creativecommons.org/licenses/by/4.0), which permits unrestricted use, distribution, and reproduction in any medium, provided the original work is properly credited. The Creative Commons Public Domain Dedication waiver (http://creativecommons.org/publicdomain/zero/1.0/) applies to the data made available in this article unless otherwise stated. 
Neuroimaging research has provided converging evidence that OCD symptoms arise from alterations in widespread brain networks. Abnormalities in frontal-striatal-thalamocortical loops are central to prevailing conceptual models of the disorder $[7,8]$. Pathological obsessions and compulsions are proposed to involve insufficient inhibitory control of striatal and thalamic nuclei by prefrontal cortical regions, particularly the anterior cingulate and lateral orbitofrontal cortices [9], and neuroimaging findings of altered regional brain volumes and function largely support these models (e.g., [10,11]). In addition, there is growing evidence implicating brain regions outside of the frontalsubcortical loops, including the posterior parietal and occipital regions [12]. Similarly, neuropsychological findings identify deficits across multiple domains of cognitive functioning, including set shifting, response inhibition, memory, and attention [13-17].

Most of the imaging literature to date is in adult rather than pediatric OCD. This is especially the case for studies that have used diffusion tensor imaging (DTI) to identify changes in brain white matter (WM) [18]. DTI is a magnetic resonance method that estimates the magnitude and direction of water diffusion, which is dependent on the underlying structure of brain tissue. In particular, highly organized myelinated axon fibers will constrain and direct water diffusion, such that DTI parameters allow inferences about WM organization and integrity. Mean diffusivity (MD) is the DTI parameter that reflects magnitude of diffusion at each voxel, which varies with tissue density regardless of fiber orientation [19]. Fractional anisotropy (FA) is the DTI parameter reflecting directionality of diffusion in each voxel, such that FA is higher along fiber bundles that are more coherent or organized [20]. FA can also be separated into components reflecting diffusion parallel and perpendicular to the WM tracts, referred to as the axial diffusivity (AD) and radial diffusivity (RD), respectively. FA values decrease when $\mathrm{AD}$ decreases and/or $\mathrm{RD}$ increases. Interestingly, RD and AD may be biomarkers of different cellular developmental or pathological processes. Specifically, changes in RD appear to be associated with cell membrane alterations (myelination), whereas variations in $\mathrm{AD}$ may be more related to axonal injuries (volume and organization) [21].

In DTI studies of adult OCD, FA has been the most commonly studied diffusion parameter. FA alterations have been observed most consistently in the corpus callosum (CC), cingulum bundle, internal capsule, and anterior thalamic radiation, as well as the parietal cortex (e.g., [22-25]). However, the directionality of FA alterations has varied, with reports of lower [23,24,26,27], higher [25,28], or comparable FA values when comparing adult OCD patients with healthy control (HC) subjects $[22,29,30]$. These seemingly disparate findings likely reflect true regional brain variability in hypo- and hyper-connectivity across fiber tracts implicated in OCD, as well as methodological differences across studies. For instance, although all studies have reported on FA, fewer have incorporated other diffusivity measures that can help contextualize FA findings. In addition, findings may relate to certain patient variables, such as psychiatric comorbidity, illness duration, and medication use. Notably, investigation of childhood OCD can help reduce some of these confounding influences including those related to illness chronicity [6] and can also isolate neurobehavioral features that relate to an early age at onset. Most adult OCD studies have combined patients with childhood and adult onset, which may introduce neurobiological variability. For instance, whereas meta-analyses of functional imaging findings indicate a central role of the caudate nucleus in adult OCD, neuroimaging studies of pediatric OCD point to more prominent involvement of other basal ganglia structures and the thalamus [5]. Thus, age at onset may be associated with neurobiological variability in OCD and could help parse disease heterogeneity.

As in the adult literature, studies of pediatric OCD have provided evidence of diffusion abnormalities in multiple WM tracts, although the nature and directionality of diffusion changes have varied, as have their associations with clinical features of illness. Two pediatric OCD DTI studies have reported FA as the main measure of diffusion [16,31]. Zarei et al. [31] found increased FA across a number of WM tracts in adolescent OCD patients, changes more widespread than those found in many adult OCD studies. Moreover, OCD symptom severity was positively correlated with FA in several of these tracts. Similarly, Gruner and colleagues [16] found that children with OCD had FA increases, though these were localized to four WM tracts, namely the left dorsal cingulum bundle, splenium of the $\mathrm{CC}$, right corticospinal tract, and left inferior fronto-occipital fasciculus. Interestingly, increased FA in the cingulum bundle predicted better executive functioning within the OCD group, suggesting that it may reflect a compensatory process. Another two pediatric OCD studies found no group differences in FA, but examination of $\mathrm{AD}$ and $\mathrm{RD}$ revealed significant diffusivity differences $[32,33]$. Specifically, Silk et al. [33] found lower AD in the genu and splenium of the CC of children with OCD compared with controls, and lower AD correlated with greater severity of symptoms. In contrast, Jayarajan et al. [32] found significantly higher $\mathrm{AD}$ and RD, and neither was significantly correlated with symptom severity, medication dosage, or treatment duration. No pediatric OCD study has yet reported on whether diffusion changes are significantly associated with age at onset.

In this study, we used DTI to compare brain WM microstructure in youth with OCD compared with healthy matched controls, examining four diffusion parameters (MD, FA, AD, and RD). We hypothesized that pediatric 
OCD would be associated with altered FA, without an priori prediction concerning the directionality of alteration (i.e., increased or decreased) due to scant published data in pediatric samples and inconsistent adult OCD DTI findings. Based on theoretical and empirical indications that age at onset may be relevant to the variability of DTI findings in OCD, we also tested the hypothesis that diffusion differences would be associated with age at onset of OCD.

\section{Methods}

\section{Subjects}

Thirty-six youth were enrolled in this neuroimaging experiment as paid volunteers. OCD subjects were treatmentseeking children presenting to an OCD clinic, and control subjects were recruited in the surrounding Boston metropolitan community via advertisements. The subjects were selected to be between 10 and 19 years old, similar to the age range in prior imaging studies of pediatric OCD. Participants were excluded if they reported current medical or neurological illness, or a history of head injury with loss of consciousness. Prior to enrollment, written informed consent was obtained from a parent/legal guardian and written informed assent was obtained from the child/ adolescent participant. All study procedures were performed in accordance with the Human Research Committees at McLean Hospital and Partners Healthcare System.

The Kiddie Schedule for Affective Disorders and Schizophrenia (KSADS) was administered to all participants and their parents by doctoral-level psychologists [34]. Individuals included in the OCD group met DSM-IV criteria for this disorder based on the KSADS [1]. In addition, OCD symptom severity scores were determined using the Children s Yale-Brown Obsessive-Compulsive Scale (CY-BOCS) [35]. To recruit a representative and generalizable OCD sample, the inclusion criteria for the OCD group allowed for comorbid psychiatric disorders, with the exception of psychotic disorders, bipolar disorder, mental retardation, substance use disorders, and pervasive developmental disorders. Neuroleptic and anti-hypertensive medications were exclusionary. All individuals included in the $\mathrm{HC}$ group were free from any current Axis I psychiatric disorder and psychotropic medications. All subjects completed the Child Depression Inventory (CDI) [36], and the Yale Global Tic Severity Scale (YGTSS) was used to rule out subjects with Tourettes syndrome and other tic-related disorders [37].

The participants consisted of 17 children with OCD and $19 \mathrm{HC}$ youth. This sample was obtained after excluding data from participants for excessive head movement (1 OCD) and poor head coverage (1 HC). The patient group endorsed the following types of OCD symptoms across previously identified clusters [38]: contamination/washing
$(N=4) ; \quad$ symmetry/arranging/counting/repeating $(N=10)$; and aggression, sexual, religious, and/or somatic obsessions/ checking $(N=14)$. No hoarding symptoms were reported. Nine (9) of the patients had no comorbid Axis I diagnosis. The following comorbidities were present in the remaining eight OCD patients, based on KSADS interviews: generalized anxiety disorder $(N=1)$, specific phobia $(N=2)$, agoraphobia $(N=1)$, major depressive disorder $(N=2)$, depression not otherwise specified $(N=2)$, oppositional defiant disorder $(N=1)$, and attention-deficit hyperactivity disorder $(N=2)$. Three (3) of the OCD patients were not taking any psychotropic medication. Primary medications taken by the remaining $14 \mathrm{OCD}$ subjects were antidepressants: selective serotonin reuptake inhibitors $(N=13)$ and tricyclic agents $(N=1)$. In addition, some patients were taking secondary medications, namely stimulants $(N=4)$, mood stabilizers $(N=3)$, and benzodiazepines $(N=1)$.

\section{Diffusion tensor imaging Image acquisition}

DTI scans were acquired using a Siemens Tim Trio 3T scanner at the McLean Hospital Imaging Center. Diffusion weighted imaging data were obtained in 60 directions with the following parameters: echo time $=98 \mathrm{~ms}$, bandwidth $=$ $1,396 \mathrm{~Hz} /$ pixel, matrix $=128 \mathrm{~mm} 128 \mathrm{~mm}, \mathrm{FOV}=$ $256 \mathrm{~mm} 256 \mathrm{~mm}, \mathrm{NEX}=1$, voxel size $=2.0 \mathrm{~mm}^{3}$ $2.0 \mathrm{~mm}^{3} 2.0 \mathrm{~mm}^{3}, 10 \mathrm{~T} 2$ low $b\left(b=0 \mathrm{~s} / \mathrm{mm}^{2}\right)$, and 60 DWI (diffusion sensitivity $b=700 \mathrm{~s} / \mathrm{mm}^{2}$ ), and 60 axial slices with 2-mm thickness.

\section{Image processing and analysis}

The analysis of DTI data was done using the FMRIB Diffusion Toolbox from the FSL processing software package (http://www.fmrib.ox.ac.uk/fsl) [39,40]. The first step was motion and eddy current distortion correction, applied using FSLs eddy_correct tool, which ran with its default options. The raw data were skull-stripped using FSLs Brain Extraction Tool (BET) [41]. A diffusion tensor model was fit at each voxel using a least squares fit to the diffusion signal with FSLs dtifit tool; this generated maps for each of the diffusivity measures (FA, MD, AD, and RD). At this point, a mathematical correction for systematic vibration artifact was applied [42]. Voxel-wise processing of diffusivity measures was carried out using tract-based spatial statistics (TBSS) [43], which is part of FSL [44]. Images from all subjects were aligned to each other using nonlinear registration in order to determine the most representative individual (i.e., the closest to the mean of the group) to be defined as the target image. This target image was then aligned, using affine registration, to MNI152 standard space. Each individual subject was then registered into the Montreal Neurological Institute (MNI) space by combining the nonlinear transform (generated 
via FSLs FNIRT) from the subject to the target image with the affine transform from the target image into the MNI space. A mean FA image was created by averaging all aligned FA maps and was thresholded with an FA $\geq 0.2$ to generate a mean FA skeleton, which represents the centers of all fiber tracts common to all subjects. Each subjects aligned FA image was projected onto the mean FA skeleton and served as the input to the TBSS. Group statistical analysis was then conducted only on voxels within the white matter skeleton mask, therefore restricting the voxel-wise analysis only to voxels with high confidence of lying within equivalent major white matter pathways in each individual. After completing the above procedures for FA, the nonlinear warps and skeleton projection were applied to MD, RD, and AD using tbss_non_FA. Differences in FA, MD, and axial and radial diffusivity between the OCD and control groups were assessed using voxelwise independent two-sample $t$-tests by randomization, the nonparametric analysis tool in FSL. The Threshold-Free Cluster Enhancement (TFCE [45]) option was employed at family-wise error-corrected $p<0.05$ to obtain cluster inferences.

\section{Statistical analyses}

Group differences in demographic characteristics were examined using $\chi^{2}$ tests for categorical variables and independent $t$-tests for continuous variables. DTI parameters (FA, RD, MD, and AD) were analyzed using permutation testing and TFCE in FSL by applying an independent $t$-test to the data for between-group comparisons. The significance level was $p<0.05$, family-wise error-corrected. Within significant clusters, the mean values were computed. Correlations of DTI variables with demographic and clinical variables were performed in SPSS version 20.

\section{Results}

Demographic and clinical data are summarized in Table 1. CY-BOCS total scores ranged from 8 to 32, spanning the mild to extreme severity range of obsessive

\begin{tabular}{llll}
$\begin{array}{l}\text { Table 1 Demographic and clinical characteristics of the } \\
\text { sample (mean SD or }\end{array}$ & $\boldsymbol{N}(\%))$ \\
\hline Variable & OCD $(\boldsymbol{n}=\mathbf{1 7})$ & Controls $(\boldsymbol{n}=\mathbf{1 9 )}$ & $\boldsymbol{p}$ \\
\hline Female & $6(35 \%)$ & $6(32 \%)$ & 0.8 \\
Age (years) & 14.062 .56 & 13.582 .12 & 0.54 \\
Education (years) & 8.652 .40 & 8.371 .95 & 0.70 \\
CDI (total) & 8.947 .16 & 3.473 .79 & $<0.01$ \\
CY-BOCS (total) & 17.068 .17 & - & \\
Obsessions & 8.124 .47 & - & \\
Compulsions & 8.944 .19 & - & \\
Age at onset (years) & 8.823 .36 & - & \\
Duration of illness (years) & 5.242 .97 & - & \\
\hline
\end{tabular}

and compulsive symptoms. In addition, OCD patients endorsed significantly higher levels of depression than $\mathrm{HC}$ subjects on the CDI $(p \mathrm{~s}<0.01)$.

\section{Between-group differences in diffusion}

OCD youth had significantly lower FA than HC subjects in widespread areas across seven separate clusters, numbered 17 in Table 2 and Figures 1 and 2. Nearly 98\% of the significant voxels localized to clusters 1 and 2 . Cluster 1 was a bilateral cluster (9,022 voxels) encompassing areas of the frontal lobes and CC (genu, body, and splenium). Cluster 2 (1,454 voxels) encompassed areas of the anterior cingulate cortex and extended into several subcortical regions including the putamen, amygdala, and thalamus. Cluster 3 (132 voxels) localized to the right angular and lateral occipital gyri. Cluster 4 (50 voxels) was in the right inferior frontal cortex, more specifically the subcallosal cortex. Cluster 5 was also a right inferior frontal cluster but more anteriorly, corresponding to the orbitofrontal cortex. Cluster 6 corresponded to 14 voxels in the right thalamus, and cluster 7 comprised 6 voxels in the right caudate and anterior internal capsule. There were no significant clusters where FA was higher in OCD patients than in $\mathrm{HC}$ subjects. Compared with $\mathrm{HC}$ subjects, OCD patients also had significantly increased RD in areas of the right frontal cortex and body of the $\mathrm{CC}$, across three clusters that overlapped with the first FA cluster (Table 3; Figure 3). There were no significant clusters where RD was lower in OCD patients than in $\mathrm{HC}$ subjects. There were no statistically significant group differences in MD or AD.

Post hoc analyses showed that the mean FA and RD of clusters that differed between the diagnostic groups did not differ significantly between OCD patients with and without current comorbid disorders.

\section{Clinical correlates of diffusion changes Age at onset}

To examine relationships with age at onset, the mean FA and mean RD were extracted from the seven FA clusters and the three RD clusters characterized above. Lower age at onset was associated with significantly decreased FA in FA cluster 6 (right thalamus: Figure 2), $r(15)=0.691, p=$ 0.002 , and with significantly increased RD in RD cluster 2 (right body of the CC), $r(15)=0.552, p=0.022$. The correlation between age at onset and FA in the thalamus remained significant after partialing out age, $r(14)=0.636$, $p=0.008$, though the correlation between age at onset and $\mathrm{RD}$ in the right body of the CC was reduced to a trend level after partialing out age, $r(14)=0.435, p=0.093$. After partialing out duration of illness, the correlations between both DTI variables and age at onset remained significant: for the FA cluster, $r(14)=0.556$ and $p=0.022$; for the RD cluster, $r(14)=0.520$ and $p=0.039$. 
Table 2 White matter clusters with reduced fractional anisotropy (FA) in pediatric OCD patients versus healthy controls

\begin{tabular}{|c|c|c|c|c|c|c|c|}
\hline \multirow[t]{2}{*}{ Cluster label and anatomical localization } & \multirow[t]{2}{*}{ Voxels } & \multicolumn{3}{|c|}{ MNI coordinates } & \multicolumn{3}{|l|}{ FA } \\
\hline & & $x$ & $y$ & $z$ & OCD & $\mathrm{HC}$ & $p$ \\
\hline $\begin{array}{l}\text { 1. Bilateral frontal (ATR, UF, IFOF, forceps minor, anterior corona radiata) } \\
\text { and corpus callosum (genu, body, splenium) }\end{array}$ & 9,022 & 20 & 37 & 6 & 0.5330 .191 & 0.5730 .188 & 0.026 \\
\hline $\begin{array}{l}\text { 2. Right cingulate and basal ganglia: anterior cingulate cortex (UF), anterior } \\
\text { limb of IC (ATR), putamen (UF), amygdala (ILF), cerebral peduncle (CST), } \\
\text { thalamus, and posterior limb of IC (ATR) }\end{array}$ & 1,454 & 23 & 0 & -9 & 0.4190 .157 & 0.4600 .158 & 0.035 \\
\hline 3. Right posterior cerebral cortex: lateral occipital and angular gyri (SLF) & 132 & 36 & -56 & 33 & 0.4230 .100 & 0.5000 .098 & 0.048 \\
\hline 4. Right inferior frontal lobe (subcallosal cortex) & 50 & 8 & 21 & -19 & 0.2960 .067 & 0.3700 .107 & 0.049 \\
\hline 5. Right inferior frontal: orbitofrontal cortex & 36 & 8 & 37 & -22 & 0.2670 .067 & 0.3230 .073 & 0.050 \\
\hline 6. Right thalamus & 14 & 18 & -5 & 11 & 0.6390 .041 & 0.6680 .038 & 0.050 \\
\hline 7. Right caudate, anterior IC (ATR) & 6 & 22 & -4 & 24 & 0.4300 .056 & $\begin{array}{ll}0.493 & 0.081\end{array}$ & 0.050 \\
\hline
\end{tabular}

ATR anterior thalamic radiation, CST corticospinal tract, IC internal capsule, IFOF inferior fronto-occipital fasciculus, ILF inferior longitudinal fasciculus, SLF superior longitudinal fasciculus, UF uncinate fasciculus.

\section{Symptom severity}

The mean FA and mean RD from the identified clusters were also examined in relation to symptom severity. There were no significant correlations with CY-BOCS total scores, CY-BOCS compulsive symptom scores, or CYBOCS obsessive symptom scores. CY-BOCS total scores were not significantly correlated with age, age at onset, or duration of illness.

\section{Discussion}

These findings add to emerging evidence of abnormal integrity of brain WM tracts in pediatric OCD. This is the first DTI study to report that children with OCD compared with healthy youth show regional brain reductions in FA. Over $84 \%$ of the significant voxels localized to a single cluster that encompassed a large expanse of bilateral frontal cortex and extended into the CC. Smaller clusters of FA reduction were seen in the right posterior parietal and occipital cortices and the subcallosal and orbitofrontal cortices, as well as the thalamus and putamen. Youth with OCD also had significantly increased RD in areas that overlapped with the largest cluster of FA reduction, including the right anterior cingulate cortex and right body of the $\mathrm{CC}$, suggesting deficient myelination in these areas. Finally, an earlier onset of OCD was associated with more pronounced FA reductions in the right thalamus and greater RD increases in the right body of the CC. Overall, our results are in line with increasing evidence that brain WM alterations are present in pediatric OCD and support a possible moderating role of age at onset on some aspects of OCD pathophysiology.

We found prominent alterations of frontal WM in subjects with OCD, affecting fiber tracts that connect the frontal lobe to both cortical and subcortical regions. Thus, OCD youth exhibited lower FA in a large cluster of bilateral frontal cortex, including anterior cingulate cortex (BA 32) and orbital-frontal cortex (BA 11), areas that are considered central to OCD pathophysiology [4,7]. This cluster overlapped with multiple WM tracts, including projection and association fibers that extend to the thalamus, anterior temporal-limbic regions, and parietal and occipital cortices. Consistent with frontal-subcortical models of OCD, FA reductions were also seen in the putamen, caudate, and thalamus of OCD youth. In adult DTI studies, both increased and decreased frontal FA have been found in OCD (e.g., $[22,24]$ ) and this may relate in part to illness heterogeneity. One source of variance in DTI studies of adult OCD appears to be the relative predominance of genetic versus environmental etiologic factors [29]. In their adult sample of monozygotic twins concordant and discordant for OCD, den Braber et al. [29] found that some WM tracts (including frontal tracts) show FA alterations that are in the opposite direction in subjects at high genetic risk compared with subjects at high environmental risk for OCD. Although neither the

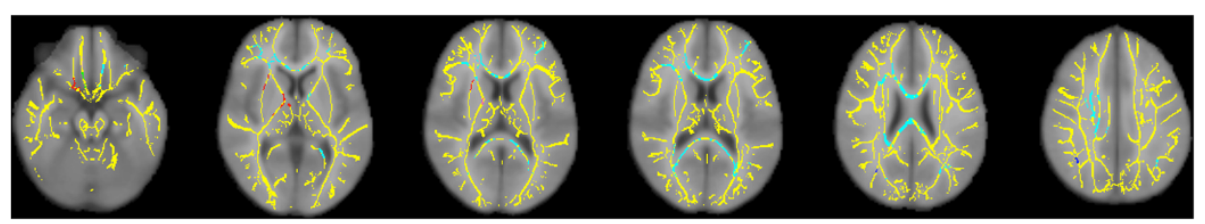

Figure 1 Clusters of significantly lower fractional anisotropy in youth with obsessive-compulsive disorder compared with healthy controls (skeleton: yellow; cluster 1: light blue; cluster 2: red; cluster 3: dark blue; cluster 4: bright green; cluster 5: copper; cluster 6: magenta; cluster 7: light green). 


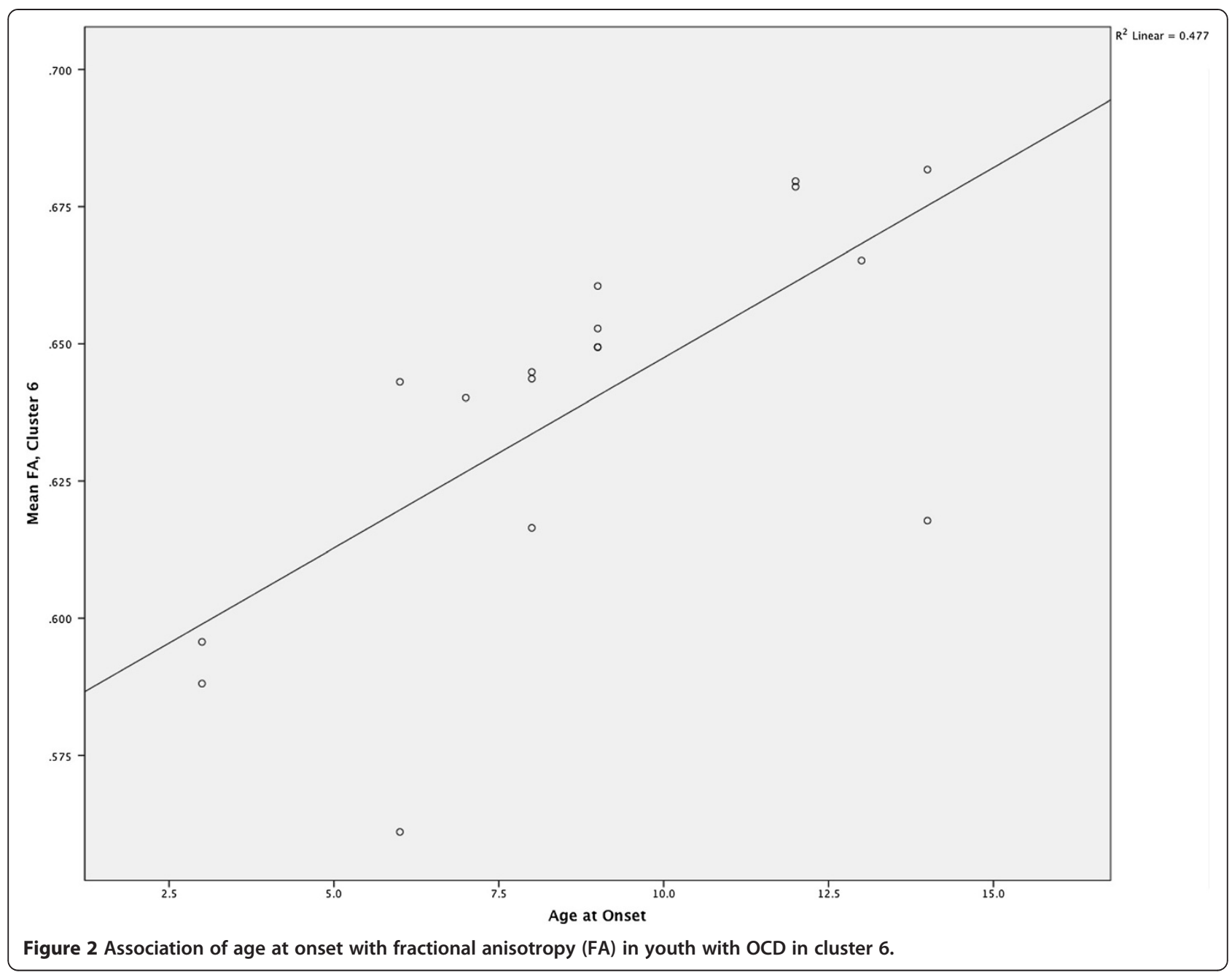

current nor prior DTI studies of pediatric OCD formally assessed family history, it is possible that our sample was relatively high on genetic loading given its particularly early age at onset (averaging several years earlier than in prior studies [31,32]) and that this would explain findings of reduced FA in our sample but not prior pediatric OCD studies. Future DTI studies could be designed to parse the influences of genetic and environmental influences on WM in pediatric OCD.

Consistent with a growing body of neurobehavioral evidence that posterior association cortices are implicated in
OCD, we found reduced WM integrity in the angular and lateral occipital gyri. A number of functional imaging studies have found abnormalities of glucose metabolism, cerebral blood flow, and brain activation in the posterior cortices of adult OCD patients [12]. Neuropsychological studies have shown that adults with OCD are impaired on visuospatial and decision-making functions that rely on the integrity of the parietal lobe [46-48]. In addition, a magnetic resonance spectroscopy study found increased choline in the parietal lobe WM of OCD, indicative of increased phospholipid turnover of myelinated axons in this

Table 3 White matter clusters with increased radial diffusivity (RD) in pediatric OCD patients versus healthy controls

\begin{tabular}{|c|c|c|c|c|c|c|c|}
\hline \multirow[t]{2}{*}{ Cluster label and anatomical localization } & \multirow[t]{2}{*}{ Voxels } & \multicolumn{3}{|c|}{ MNI coordinates } & \multicolumn{3}{|l|}{ RD } \\
\hline & & $x$ & $y$ & $z$ & OCD & $\mathrm{HC}$ & $p$ \\
\hline $\begin{array}{l}\text { 1. Right frontal (anterior corona radiata, forceps minor, } \\
\text { ATR; overlaps FA cluster 1) }\end{array}$ & 406 & 19 & 32 & 12 & 0.0006550 .000085 & 0.0005940 .000089 & 0.042 \\
\hline 2. Right body of the corpus callosum (overlaps FA cluster 1) & 81 & 12 & 4 & 28 & 0.0004210 .000085 & 0.0003580 .000083 & 0.049 \\
\hline $\begin{array}{l}\text { 3. Right body of the corpus callosum (close to midline; } \\
\text { overlaps FA cluster 1) }\end{array}$ & 49 & 3 & 4 & 23 & 0.0004740 .000084 & 0.0003990 .000077 & 0.049 \\
\hline
\end{tabular}




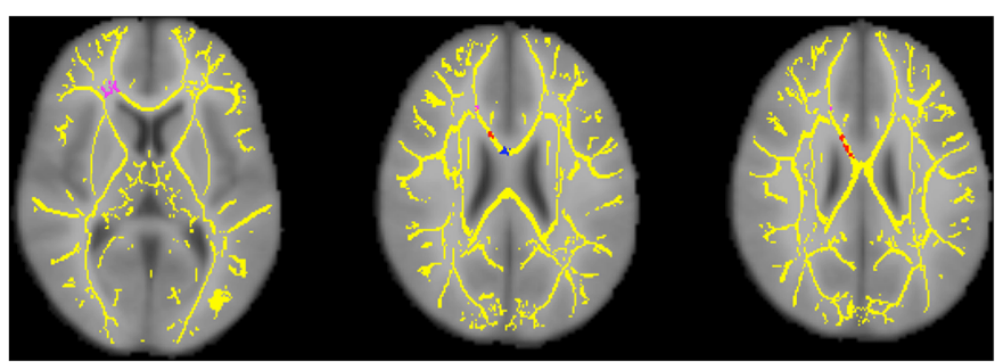

Figure 3 Clusters of significantly higher radial diffusivity in youth with obsessive-compulsive disorder compared with healthy controls (skeleton: yellow; cluster 1: magenta; cluster 2: red; cluster 3: blue).

region [49]. Although prior childhood OCD DTI studies found evidence of $\mathrm{CC}$ alterations suggestive of posterior association cortex involvement, the current study is the first to identify a cluster of altered FA in parietal cortex of pediatric patients. In DTI studies of adult OCD, reduced FA in parietal WM also has been found to distinguish patients from healthy control subjects (e.g., [12,24]). Thus, our results extend those of adult DTI studies by indicating that reduced parietal WM integrity is present in pediatric patients. This finding converges with a report of reduced parietal WM volume in adolescents with OCD compared with healthy youth [50]. Neurodevelopmental studies have shown that increases in FA and other maturational changes occur in parietal-occipital WM structure from the earliest years of childhood [51], such that disruption of this process could relate to early-onset pathophysiology [52]. In adult studies, visuospatial deficits and intrusive visual imagery are prominent features of OCD and have been documented premorbidly in adults prior to their onset of OCD symptoms [53]. Altogether, this suggests that abnormal parietal-occipital lobe structure and its behavioral manifestations are present in childhood OCD and early in the course of adult OCD.

Findings of diffusion alterations in the $\mathrm{CC}$ add to growing evidence of interhemispheric abnormalities in OCD, evident in both the pediatric and adult literatures $[18,54]$. In this study, OCD compared with healthy youth had reduced FA in portions of the genu, body, and splenium, which implicates fibers that connect bilateral frontal, parietal, and temporal-occipital association cortices $[55,56]$. There was a prominent cluster of FA reduction in the middle to posterior body of the $\mathrm{CC}$, which connects bilateral somatosensory cortices and posterior parietal regions [57]. We also found decreased FA in the anterior portion of the genu, where small axons connect bilateral prefrontal cortex and ventral prefrontal cortex to the striatum. In an earlier study of pediatric OCD, Zarei et al. [31] had found increased FA in somewhat different CC areas corresponding to the posterior genu and anterior body of the $\mathrm{CC}$, where larger axons connect primary motor cortices. Thus, it is possible that directional coherence of fiber tracts is differentially altered across different sections of the genu and body of the CC in childhood OCD, indicating variable pathology across topographically distinct association cortices.

Our finding of decreased splenium FA conflicts with two prior reports on youth with OCD [16,31]. Both investigations found higher splenium $\mathrm{FA}$, which correlated with significantly greater OCD symptom severity [16,31]. Both of these prior studies had a lower proportion of medicated OCD patients $(61 \%, 52 \%)$ than the current study (82\%). Moreover, significantly elevated splenium FA was seen only among the subgroup of unmedicated patients in one of those studies [16]. Thus, it may be that our finding of lower splenium FA reflects the effects of medication treatment. Our study design does not allow us to test this question, but there is some prior evidence that treatmentnave OCD patients, including pediatric patients, have higher FA, greater WM density, and larger size of the CC [57]. Moreover, in a longitudinal study of adults with OCD, Yoo et al. [25] found that drug-nave patients had multiple areas of increased FA (including in the posterior $\mathrm{CC}$ ) and that these normalized after a course of clinically effective citalopram treatment. Another possible source of conflicting findings across DTI studies is heterogeneity of OCD clinical features (e.g., our sample included a minority of washers relative to checkers ). The adult OCD literature has begun to explore neural correlates of symptom dimensions in OCD [58]. Similar studies could be designed in pediatric OCD by selecting more homogeneous patient samples.

Alterations in diffusion anisotropy (FA) can result from changes in either RD (perpendicular) and/or AD (parallel), and these subcomponents are differentially modulated by myelin and axonal degeneration mechanisms, respectively [59]. In this study, we found concomitant decreases in FA and increases in RD in the right frontal cortex and right body of the CC, a pattern thought to reflect deficient myelination [59-62]. Abnormal myelination also has been implicated in OCD by other lines of research, including magnetic resonance spectroscopy findings of increased levels of cell membrane breakdown products in OCD 
youth [63,64], and genetic evidence of an association between OCD and a gene (OLIG2) involved in the development of oligodendrocytes [65]. Similar to our study, Jayarajan and colleagues [32] also had found significantly increased RD in a DTI in children with OCD compared with controls, although FA did not differ significantly between groups. Because increased RD was accompanied by increased AD in their patient sample, Jayarajan et al. [32] interpreted this as indicative of hyperconnected yet insufficiently myelinated WM tracts in affected regions. In contrast, Gruner et al. [16] found decreased RD in the context of significantly increased FA in four WM regions of pediatric OCD compared with control subjects, pointing to excessive myelination of certain axon fibers. Finally, Silk et al. [33] found significantly decreased AD in the genu and splenium of the CC of OCD youth, which could indicate less coherently organized callosal axons; FA was not significantly different between the groups in the CC or any other WM tract of that study. Thus, in the relatively small literature to date, there are different patterns of FA/RD/AD findings across pediatric OCD DTI studies. This could indicate involvement of multiple possible combinations of WM microstructure alterations in pediatric OCD wherein both myelin and axonal changes may be present in the context of either reduced or increased coherence of fiber tracts. The pattern of abnormalities may vary across brain regions, due to age-related maturational processes [16,31] and/or as a function of clinical phenomenology. These questions will need to be disentangled by conducting additional studies in this relatively young area of study.

Our findings suggest that age at onset may be an influential moderating factor on some of the WM changes in pediatric OCD. On average, our patients had an earlier onset of illness (average 8 years) than those in the two earlier pediatric OCD studies that reported on age at onset (11 years [31] and 13 years [32] on average). Moreover, within our patient sample, earlier onset was associated with significantly lower FA in the right thalamus and significantly higher $\mathrm{RD}$ in the right $\mathrm{CC}$. The former association remained significant even after controlling for age, suggesting that earlier onset of OCD disease-related processes is associated with reduced integrity of WM in the thalamus. Involvement of the thalamus in early-onset OCD is consistent with the conclusions of a literature review that prominent thalamic abnormalities may be a component of a slightly different neuropathological substrate of childhood-onset versus adult-onset OCD [54].

Studying pediatric patients near the onset of their illness helps identify neurobiological changes that may be more primary than those arising later on in the course of illness, although developmental and disease effects may still be influential. Brain alterations noted during childhood and adolescence may change in their nature due to ongoing maturation of both gray matter and WM, which may contribute to variable findings across studies. In addition, compulsively engaging in a particular type of behavior or cognitive process can change brain structure $[4,66,67]$. Thus, even in pediatric OCD, anatomical brain differences may reflect a consequence rather than a precursor of the disorder. In the only study of neuropsychological correlates of DTI measures in pediatric OCD, the pattern of results found by Gruner et al. [16] suggested that certain FA increases might reflect compensatory increases in WM coherence to mitigate neuropsychological deficits. This highlights the usefulness of studying patients with OCD as close to symptom onset as possible, when such epiphenomenal changes may be less prominent.

Our findings should be interpreted in the context of the studys limitations. This was a cross-sectional investigation, and our results are therefore correlational in nature. We cannot rule out that these findings were affected by comorbidity within our OCD sample, particularly depression and other anxiety disorders present in a third of the patients. However, in post hoc analyses, severity of depression and anxiety symptoms was not associated with FA or $\mathrm{RD}$ in any of the significant clusters. Moreover, only a small percentage of the sample had any particular comorbid condition, making it implausible that the group findings were driven by a particular comorbidity. Similarly, we cannot exclude the possibility of medication effects, since most of our patients were medicated; although none of the previous pediatric OCD studies found any indication that DTI parameters were affected by medication use $[16,31,32]$, it is possible that this reflected limited power to detect such effects. Finally, this and other pediatric OCD studies to date have not had sufficient power to examine the relationship of DTI WM measures with OCD symptom profile, which have been found to differentially relate to regional brain abnormalities in adult OCD [68].

\section{Conclusions}

Our findings of significantly lower FA and higher RD in childhood OCD are consistent with compromised WM integrity and reduced myelination, particularly involving the $\mathrm{CC}$ and fiber tracts that connect the frontal lobes to widespread cortical and subcortical targets. Our findings also suggest that age at onset may be a moderator of some of the WM changes in children with OCD. DTI research in childhood OCD is still in its infancy, and future studies should incorporate examination of how genetic and environmental risk factors, as well as aspects of illness phenomenology, may help parse divergent findings across studies.

\section{Abbreviations}

AD: Axial diffusivity; CC: Corpus callosum; CDI: Child Depression Inventory; CY-BOCS: Children s Yale-Brown Obsessive-Compulsive Scale; DTI: Diffusion tensor imaging; FA: Fractional anisotropy; HC: Healthy control; KSADS: Kiddie 
Schedule for Affective Disorders and Schizophrenia; MD: Mean diffusivity; RD: Radial diffusivity; OCD: Obsessive-compulsive disorder; TBSS: Tract-based spatial statistics; TFCE: Threshold-Free Cluster Enhancement; WM: White matter; YGTSS: Yale Global Tic Severity Scale.

\section{Competing interests}

The authors declare that they have no competing interests.

\section{Authors contributions}

IMR was involved in data collection and analysis and wrote the first draft of the manuscript. EAO analyzed the imaging data and contributed to writing of the methods and results. JCB, SES, and SLR designed the study and were involved in subject recruitment and data interpretation. GP and NM contributed to the processing, analysis, and interpretation of the imaging data. WDSK was involved in data collection. SW and MAJ assisted with subject recruitment. All authors contributed to and have approved the final manuscript.

\section{Acknowledgements}

This research was supported by an anonymous donor. We would like to acknowledge the contributions of Jennifer Ragan, Anne Chosak, Adriane Alpern, Elizabeth Flamm, Sarah Glaser, and Elizabeth Sadock for their assistance in conducting assessments and the McLean Hospital Imaging Center MRI technologists for their assistance with scanning.

\section{Author details}

${ }^{1}$ Center for Depression, Anxiety and Stress Research, McLean Hospital, 115 Mill Street, mailstop 334, Belmont, MA 02478, USA. ${ }^{2}$ Department of Psychiatry, Harvard Medical School, Boston, MA, USA. ${ }^{3}$ Department of Psychology, University of Miami, 5665 Ponce de Leon Blvd, Flipse Building, Coral Gables, Miami, FL 33146, USA. ${ }^{4}$ British Columbia Mental Health and Addictions Research Institute, University of British Columbia, Vancouver, BC, Canada. ${ }^{5}$ Center for Morphometric Analysis, Massachusetts General Hospital, Bldg 149, 13th Street, Charlestown, MA 02129, USA. ${ }^{6}$ Department of Neurology, Harvard Medical School, Boston, MA, USA. ${ }^{7}$ Department of Radiology, Harvard Medical School, Boston, MA, USA. ${ }^{8}$ Department of Psychiatry, Massachusetts General Hospital, Simches Research Building, 185 Cambridge Street, Suite 2282, Boston, MA 02114, USA. ${ }^{9}$ UBC Psychiatry A3-118, 938 West 28th Ave, Vancouver, BC V5Z 4H4, Canada. ${ }^{10}$ Department of Psychiatry, University of Arizona, Tucson, AZ, USA.

Received: 2 July 2014 Accepted: 14 November 2014

Published online: 18 December 2014

\section{References}

1. American Psychiatric Association: Diagnostic and Statistical Manual of Mental Disorders, Fourth Edition (DSM-IV). Washington, D.C.: American Psychiatric Association; 1994.

2. Geller DA: Obsessive-compulsive and spectrum disorders in children and adolescents. Psychiatr Clin North Am 2006, 29:353 370.

3. Stewart SE, Geller DA, Jenike M, Pauls D, Shaw D, Mullin B, Faraone SV: Long-term outcome of pediatric obsessive-compulsive disorder: a meta-analysis and qualitative review of the literature. Acta Psychiatr Scand 2004, 110:4 13.

4. Maia TV, Cooney RE, Peterson BS: The neural bases of obsessive-compulsive disorder in children and adults. Dev Psychopathol 2008, 20:1251 1283.

5. Huyser C, Veltman DJ, de Haan E, Boer F: Paediatric obsessive-compulsive disorder, a neurodevelopmental disorder? Evidence from neuroimaging. Neurosci Biobehav Rev 2009, 33:818 830.

6. Rosenberg DR, Keshavan MS: A.E. Bennett Research Award. Toward a neurodevelopmental model of obsessive compulsive disorder. Biol Psychiatry 1998, 43:623 640.

7. Graybiel AM, Rauch SL: Toward a neurobiology of obsessive-compulsive disorder. Neuron 2000, 28:343 347.

8. Saxena S, Brody AL, Schwartz JM, Baxter LR: Neuroimaging and frontal-subcortical circuitry in obsessive-compulsive disorder. Br J Psychiatry Suppl 1998, 35:26 37.

9. Fineberg NA, Potenza MN, Chamberlain SR, Berlin HA, Menzies L, Bechara A, Sahakian BJ, Robbins TW, Bullmore ET, Hollander E: Probing compulsive and impulsive behaviors, from animal models to endophenotypes: a narrative review. Neuropsychopharmacology 2010, 35:591 604.
10. Radua J, van den Heuvel OA, Surguladze S, Mataix-Cols D: Meta-analytical comparison of voxel-based morphometry studies in obsessive-compulsive disorder vs other anxiety disorders. Arch Gen Psychiatry 2010, 67:701 711.

11. Rotge JY, Guehl D, Dilharreguy B, Tignol J, Bioulac B, Allard M, Burbaud P, Aouizerate B: Meta-analysis of brain volume changes in obsessive-compulsive disorder. Biol Psychiatry 2009, 65:75 83.

12. Menzies L, Chamberlain SR, Laird AR, Thelen SM, Sahakian BJ, Bullmore ET: Integrating evidence from neuroimaging and neuropsychological studies of obsessive-compulsive disorder: the orbitofronto-striatal model revisited. Neurosci Biobehav Rev 2008, 32:525 549.

13. Abramovitch A, Dar R, Schweiger A, Hermesh H: Neuropsychological impairments and their association with obsessive-compulsive symptom severity in obsessive-compulsive disorder. Arch Clin Neuropsychol 2011, 26:364 376.

14. Bannon S, Gonsalvez CJ, Croft RJ, Boyce PM: Response inhibition deficits in obsessive-compulsive disorder. Psychiatry Res 2002, 110:165 174.

15. Chamberlain SR, Blackwell AD, Fineberg NA, Robbins TW, Sahakian BJ: The neuropsychology of obsessive compulsive disorder: the importance of failures in cognitive and behavioural inhibition as candidate endophenotypic markers. Neurosci Biobehav Rev 2005, 29:399 419.

16. Gruner P, Vo A, Ikuta T, Mahon K, Peters BD, Malhotra AK, Ulug AM, Szeszko PR: White matter abnormalities in pediatric obsessive-compulsive disorder. Neuropsychopharmacology 2012, 37:2730 2739.

17. Penades R, Catalan R, Rubia K, Andres S, Salamero M, Gasto C: Impaired response inhibition in obsessive compulsive disorder. Eur Psychiatry 2007, 22:404 410

18. Fontenelle LF, Harrison BJ, Yucel M, Pujol J, Fujiwara H, Pantelis C: Is there evidence of brain white-matter abnormalities in obsessive-compulsive disorder?: A narrative review. Top Magn Reson Imaging 2009, 20:291 298

19. Olson EA, Collins PF, Hooper CJ, Muetzel R, Lim KO, Luciana M: White matter integrity predicts delay discounting behavior in 9- to 23-year-olds: a diffusion tensor imaging study. J Cogn Neurosci 2009, 21:1406 1421.

20. Beaulieu C: The basis of anisotropic water diffusion in the nervous system - a technical review. NMR Biomed 2002, 15:435 455.

21. Harsan LA, Poulet P, Guignard B, Steibel J, Parizel N, de Sousa PL, Boehm N, Grucker D, Ghandour MS: Brain dysmyelination and recovery assessment by noninvasive in vivo diffusion tensor magnetic resonance imaging. J Neurosci Res 2006, 83:392 402

22. Cannistraro PA, Makris N, Howard JD, Wedig MM, Hodge SM, Wilhelm S, Kennedy DN, Rauch SL: A diffusion tensor imaging study of white matter in obsessive-compulsive disorder. Depress Anxiety 2007, 24:440 446.

23. Garibotto V, Scifo P, Gorini A, Alonso CR, Brambati S, Bellodi L, Perani D: Disorganization of anatomical connectivity in obsessive compulsive disorder: a multi-parameter diffusion tensor imaging study in a subpopulation of patients. Neurobiol Dis 2010, 37:468 476.

24. Szeszko PR, Ardekani BA, Ashtari M, Malhotra AK, Robinson DG, Bilder RM, Lim KO: White matter abnormalities in obsessive-compulsive disorder: a diffusion tensor imaging study. Arch Gen Psychiatry 2005, 62:782 790.

25. Yoo SY, Jang JH, Shin YW, Kim DJ, Park HJ, Moon WJ, Chung EC, Lee JM, Kim IY, Kim SI, Kwon JS: White matter abnormalities in drug-naive patients with obsessive-compulsive disorder: a diffusion tensor study before and after citalopram treatment. Acta Psychiatr Scand 2007, 116:211 219.

26. Nakamae T, Narumoto J, Sakai Y, Nishida S, Yamada K, Nishimura T, Fukui K: Diffusion tensor imaging and tract-based spatial statistics in obsessive-compulsive disorder. J Psychiatr Res 2011, 45:687 690.

27. Saito Y, Nobuhara K, Okugawa G, Takase K, Sugimoto T, Horiuchi M, Ueno C, Maehara M, Omura N, Kurokawa H, Ikeda K, Tanigawa N, Sawada S, Kinoshita T: Corpus callosum in patients with obsessive-compulsive disorder: diffusion-tensor imaging study. Radiology 2008, 246:536 542.

28. Li F, Huang X, Yang Y, Li B, Wu Q, Zhang T, Lui S, Kemp GJ, Gong Q: Microstructural brain abnormalities in patients with obsessive-compulsive disorder: diffusion-tensor MR imaging study at 3.0 T. Radiology 2011, 260:216 223.

29. den Braber A, van t D, Boomsma DI, Cath DC, Veltman DJ, Thompson PM, de Geus EJ: White matter differences in monozygotic twins discordant or concordant for obsessive-compulsive symptoms: a combined diffusion tensor imaging/voxel-based morphometry study. Biol Psychiatry 2011, 70:969 977.

30. Menzies L, Williams GB, Chamberlain SR, Ooi C, Fineberg N, Suckling J, Sahakian BJ, Robbins TW, Bullmore ET: White matter abnormalities in 
patients with obsessive-compulsive disorder and their first-degree relatives. Am J Psychiatry 2008, 165:1308 1315.

31. Zarei M, Mataix-Cols D, Heyman I, Hough M, Doherty J, Burge L, Winmill L, Nijhawan S, Matthews PM, James A: Changes in gray matter volume and white matter microstructure in adolescents with obsessive-compulsive disorder. Biol Psychiatry 2011, 70:1083 1090.

32. Jayarajan RN, Venkatasubramanian G, Viswanath B, Janardhan Reddy YC, Srinath S, Vasudev MK, Chandrashekar CR: White matter abnormalities in children and adolescents with obsessive-compulsive disorder: a diffusion tensor imaging study. Depress Anxiety 2012, 29:780 788.

33. Silk T, Chen J, Seal M, Vance A: White matter abnormalities in pediatric obsessive-compulsive disorder. Psychiatry Res 2013, 213:154 160

34. Kaufman J, Birmaher B, Brent D, Rao U, Flynn C, Moreci P, Williamson D, Ryan N: Schedule for affective disorders and schizophrenia for school-Age children-present and lifetime version (K-SADS-PL): initial reliability and validity data. J Am Acad Child Adolesc Psychiatry 1997, 36:980 988.

35. Goodman WK, Price LH, Rasmussen SA, Mazure C, Fleischmann RL, Hill CL, Heninger GR, Charney DS: The Yale-Brown Obsessive Compulsive Scale. I. Development, use, and reliability. Arch Gen Psychiatry 1989, 46:1006 1011.

36. Kovacs M: Childrens Depression Inventory Manual. North Tonawanda: Multi-Health Systems; 1992

37. Leckman JF, Riddle MA, Hardin MT, Ort SI, Swartz KL, Stevenson J, Cohen DJ: The Yale Global Tic Severity Scale: initial testing of a clinician-rated scale of tic severity. J Am Acad Child Adolesc Psychiatry 1989, 28:566 573.

38. Stewart SE, Rosario MC, Baer L, Carter AS, Brown TA, Scharf JM, Illmann C, Leckman JF, Sukhodolsky D, Katsovich L, Rasmussen S, Goodman W, Delorme R, Leboyer M, Chabane N, Jenike MA, Geller DA, Pauls DL: Four-factor structure of obsessive-compulsive disorder symptoms in children, adolescents, and adults. J Am Acad Child Adolesc Psychiatry 2008, 47:763 772

39. Andersson JLR, Jenkinson M, Smith S: Non-linear optimisation. [www.fmrib. ox.ac.uk/analysis/techrep]

40. Andersson $J L R$, Jenkinson $M$, Smith S: Non-linear registration, aka spatial normalisation. [www.fmrib.ox.ac.uk/analysis/techrep]

41. Smith SM: Fast robust automated brain extraction. Hum Brain Mapp 2002, 17:143 155 .

42. Gallichan D, Scholz J, Bartsch A, Behrens TE, Robson MD, Miller KL: Addressing a systematic vibration artifact in diffusion-weighted MRI. Hum Brain Mapp 2010, 31:193 202.

43. Smith SM, Jenkinson M, Johansen-Berg H, Rueckert D, Nichols TE, Mackay CE, Watkins KE, Ciccarelli O, Cader MZ, Matthews PM, Behrens TE: Tract-based spatial statistics: voxelwise analysis of multi-subject diffusion data. Neuroimage 2006, 31:1487 1505.

44. Smith SM, Jenkinson M, Woolrich MW, Beckmann CF, Behrens TE, Johansen-Berg H, Bannister PR, De Luca M, Drobnjak I, Flitney DE, Niazy RK, Saunders J, Vickers J, Zhang Y, De Stefano N, Brady JM, Matthews PM: Advances in functional and structural MR image analysis and implementation as FSL. Neuroimage 2004, 23(Suppl 1):S208 S219.

45. Nichols TE, Holmes AP: Nonparametric permutation tests for functional neuroimaging: a primer with examples. Hum Brain Mapp 2002, 15:1 25.

46. Chamberlain SR, Fineberg NA, Blackwell AD, Clark L, Robbins TW, Sahakian BJ: A neuropsychological comparison of obsessive-compulsive disorder and trichotillomania. Neuropsychologia 2007, 45:654 662

47. Menzies L, Achard S, Chamberlain SR, Fineberg N, Chen CH, del Campo N, Sahakian BJ, Robbins TW, Bullmore E: Neurocognitive endophenotypes of obsessive-compulsive disorder. Brain 2007, 130:3223 3236.

48. Okasha A, Rafaat M, Mahallawy N, El Nahas G, El Dawla AS, Sayed M, El Kholi S: Cognitive dysfunction in obsessive-compulsive disorder. Acta Psychiatr Scand 2000, 101:281 285

49. Kitamura H, Shioiri T, Kimura T, Ohkubo M, Nakada T, Someya T: Parietal white matter abnormalities in obsessive-compulsive disorder: a magnetic resonance spectroscopy study at 3-Tesla. Acta Psychiatr Scand 2006, 114:101 108.

50. Lazaro L, Bargallo N, Castro-Fornieles J, Falcon C, Andres S, Calvo R, Junque C: Brain changes in children and adolescents with obsessive-compulsive disorder before and after treatment: a voxel-based morphometric MRI study. Psychiatry Res 2009, 172:140 146.

51. Giedd JN, Blumenthal J, Jeffries NO, Castellanos FX, Liu H, Zijdenbos A, Paus T, Evans AC, Rapoport JL: Brain development during childhood and adolescence: a longitudinal MRI study. Nat Neurosci 1999, 2:861 863.
52. Barnea-Goraly N, Menon V, Eckert M, Tamm L, Bammer R, Karchemskiy A, Dant CC, Reiss AL: White matter development during childhood and adolescence: a cross-sectional diffusion tensor imaging study. Cereb Cortex 2005, 15:1848 1854.

53. Grisham JR, Anderson TM, Poulton R, Moffitt TE, Andrews G: Childhood neuropsychological deficits associated with adult obsessive-compulsive disorder. Br J Psychiatry 2009, 195:138 141.

54. MacMaster FP, O Neill J, Rosenberg DR: Brain imaging in pediatric obsessive-compulsive disorder. J Am Acad Child Adolesc Psychiatry 2008, 47:1262 1272 .

55. Giedd JN, Rumsey JM, Castellanos FX, Rajapakse JC, Kaysen D, Vaituzis AC, Vauss YC, Hamburger SD, Rapoport JL: A quantitative MRI study of the corpus callosum in children and adolescents. Brain Res Dev Brain Res 1996, 91:274 280.

56. Mac Master FP, Keshavan MS, Dick EL, Rosenberg DR: Corpus callosal signal intensity in treatment-naive pediatric obsessive compulsive disorders. Prog Neuropsychopharmacol Biol Psychiatry 1999, 23:601 612.

57. Rosenberg DR, Keshavan MS, Dick EL, Bagwell WW, MacMaster FP, Birmaher B: Corpus callosal morphology in treatment-naive pediatric obsessive compulsive disorder. Prog Neuropsychopharmacol Biol Psychiatry 1997, 21:1269 1283 .

58. Mataix-Cols D, Cullen S, Lange K, Zelaya F, Andrew C, Amaro E, Brammer MJ, Williams SC, Speckens A, Phillips ML: Neural correlates of anxiety associated with obsessive-compulsive symptom dimensions in normal volunteers. Biol Psychiatry 2003, 53:482 493.

59. Song SK, Sun SW, Ramsbottom MJ, Chang C, Russell J, Cross AH: Dysmyelination revealed through $\mathrm{MRI}$ as increased radial (but unchanged axial) diffusion of water. Neuroimage 2002, 17:1429 1436.

60. Basser PJ: Inferring microstructural features and the physiological state of tissues from diffusion-weighted images. NMR Biomed 1995, 8:333 344

61. Song SK, Sun SW, Ju WK, Lin SJ, Cross AH, Neufeld AH: Diffusion tensor imaging detects and differentiates axon and myelin degeneration in mouse optic nerve after retinal ischemia. Neuroimage 2003, 20:1714 1722.

62. Song SK, Yoshino J, Le TQ, Lin SJ, Sun SW, Cross AH, Armstrong RC: Demyelination increases radial diffusivity in corpus callosum of mouse brain. Neuroimage 2005, 26:132 140.

63. Rosenberg DR, MacMillan SN, Moore GJ: Brain anatomy and chemistry may predict treatment response in paediatric obsessive compulsive disorder. Int J Neuropsychopharmacol 2001, 4:179 190.

64. Smith EA, Russell A, Lorch E, Banerjee SP, Rose M, Ivey J, Bhandari R, Moore GJ, Rosenberg DR: Increased medial thalamic choline found in pediatric patients with obsessive-compulsive disorder versus major depression or healthy control subjects: a magnetic resonance spectroscopy study. Biol Psychiatry 2003, 54:1399 1405.

65. Stewart SE, Platko J, Fagerness J, Birns J, Jenike E, Smoller JW, Perlis R, Leboyer M, Delorme R, Chabane N, Rauch SL, Jenike MA, Pauls DL: A genetic family-based association study of OLIG2 in obsessive-compulsive disorder. Arch Gen Psychiatry 2007, 64:209 214.

66. Lazar SW, Kerr CE, Wasserman RH, Gray JR, Greve DN, Treadway MT, McGarvey M, Quinn BT, Dusek JA, Benson H, Rauch SL, Moore Cl, Fischl B: Meditation experience is associated with increased cortical thickness. Neuroreport 2005, 16:1893 1897.

67. Schlaug G: The brain of musicians. A model for functional and structural adaptation. Ann N Y Acad Sci 2001, 930:281 299.

68. van den Heuvel OA, Remijnse PL, Mataix-Cols D, Vrenken H, Groenewegen HJ, Uylings $\mathrm{HB}$, van Balkom AJ, Veltman DJ: The major symptom dimensions of obsessive-compulsive disorder are mediated by partially distinct neural systems. Brain 2009, 132:853 868.

doi:10.1186/s13587-014-0013-6

Cite this article as: Rosso et al:: Brain white matter integrity and association with age at onset in pediatric obsessive-compulsive disorder. Biology of Mood \& Anxiety Disorders 2014 4:13. 